\title{
Necrotizing Otitis Externa: A Disease Barely Known to Neurosurgeons
}

\section{Otite externa necrotizante: uma doença pouco conhecida entre os neurocirurgiões}

\author{
Sérgio Augusto Vieira Cançado ${ }^{1}$ Lucídio Duarte de Souza ${ }^{1}$ Rodrigo Moreira Faleiro ${ }^{1}$ \\ José Maurício Siqueira ${ }^{1}$ \\ ${ }^{1}$ Neurosurgery Service, Clínica Neurológica e Neurocirúrgica, \\ Hospital Felício Rocho, Belo Horizonte, MG, Brazil \\ Arq Bras Neurocir 2019;38:239-245. \\ Address for correspondence Sérgio Augusto Vieira Cançado, MSc, \\ Secretaria de Neurocirurgia, Hospital Felício Rocho, Av. do Contorno, \\ 9530, $2^{\circ}$ andar, Barro Preto, Belo Horizonte, MG, 30110-934, Brazil \\ (e-mail: sergiomed124@gmail.com).
}

Necrotizing otitis externa (NOE), also known as malignant otitis externa (MOE), is a severe and rare infectious disease of the external auditory canal (EAC). Without treatment, it may progress to skull base involvement. The bacteria Pseudomonas aeruginosa is the most common causative agent ( $\sim 90 \%$ of the cases), and affects immunocompromised subjects, particularly diabetic patients. Severe chronic otalgia, otorrhea, and cranial nerve palsy are the most common clinical presentations. Patients with NOE are frequently referred to neurosurgery because of the neurological impairment and skull base compromise. The definitive diagnosis is frequently elusive, requiring a high index of suspicion. Several laboratorial tests, imaging modalities, and the histologic exclusion of malignancy may be required. An early diagnosis and aggressive treatment reduce morbidity and mortality. We present four NOE cases to illustrate the spectrum of clinical presentation and complementary exams. According to the literature, more effort for early diagnosis and treatment is required, and neurosurgeons play an important role in this task.

Otite externa necrotizante (OEN), também conhecida como otite externa maligna (OEM), é uma doença infecciosa grave do conduto auditivo externo. Sem o tratamento adequado, pode progredir com acometimento da base do crânio. A bactéria Pseudomonas aeruginosa é o agente etiológico mais comum ( $~ 90 \%$ dos casos), e afeta pacientes imunocomprometidos, principalmente diabéticos. Otalgia crônica severa, otorreia, e déficit em nervos cranianos são as apresentações clínicas mais comuns. Pacientes com OEN frequentemente são encaminhados ao neurocirurgião devido ao déficit neurológico e ao comprometimento da base do crânio. O diagnóstico é complexo e exige alto índice de suspeição. Diversos exames laboratoriais, de imagem e exclusão por histologia de neoplasia podem ser necessários. O tratamento precoce e agressivo reduz a morbimortalidade. Apresentamos quatro casos de OEN para ilustrar o espectro de apresentação clínica e exames complementares. De acordo com a literatura, é necessário mais empenho para o diagnóstico e o tratamento precoce, e o neurocirurgião tem importante papel nessas tarefas. received

July 25, 2016

accepted

October 19, 2016
DOI https://doi.org/

10.1055/s-0036-1596052. ISSN 0103-5355.
Copyright $(2019$ by Thieme Revinter

Publicações Ltda, Rio de Janeiro, Brazil
License terms

(1) (1) $\Theta \circledast$ 


\section{Introduction}

Necrotizing otitis externa (NOE) or malignant otitis externa (MOE) is an infection of the soft parts of the external auditory canal (EAC) that penetrates the skull and can affect the temporal bone and other components of the base of the skull. Its description as a clinical entity dates from 1959. ${ }^{1}$ In the 1990s, studies elucidated factors related to its pathogenesis and distinct evolution forms, such as mastoiditis and skull base osteomyelitis (SBO). ${ }^{2,3}$

The pathophysiology of NOE involves an invasive infection that begins in the EAC and penetrates the skull through the Santorini fissure, with Pseudomonas aeruginosa as the etiological agent in up to $98 \%$ of the cases. ${ }^{3}$ Immunity-impairing comorbidities (HIV, pharmacological immunosuppression, diabetes mellitus, etc.), ear irrigation for EAC cleaning, and local alterations (microangiopathy of the EAC and change in the $\mathrm{pH}$ of the cerumen in diabetic patients) are the main risk factors. ${ }^{2,3}$

The natural history of the untreated disease is the contiguous involvement of the bones from the base of the skull in several patterns, leading to cranial nerve damage.

The progression to SBO with the involvement of the temporal, occipital, and other bones, although rare, results in great morbidity and mortality. ${ }^{4}$ Skull base osteomyelitis can also be triggered by odontogenic or paranasal sinuses involvement, but less frequently when compared with SBO secondary to NOE. ${ }^{5}$

\section{Methodology}

Four cases of NOE evaluated and treated at the Neurosurgery Service of the Hospital Felício Rocho are described. A flowchart is proposed to approach and treat this condition.

\section{Case 1}

Male patient, 58 years old, with severe otalgia for 30 days associated with neck pain and right temporal headache. Previous history of insulin-dependent diabetes mellitus. Otitis for 11 months with secondary right facial paresis, treated in primary care with topical corticosteroids and oral amoxicillin for 2 weeks.

\section{Physical Exam}

Right-sided peripheral facial paresis (House-Brackmann IV). Otoscopy showed perforation of the right tympanic membrane.

\section{Adjuvant Tests}

Complete blood count showing discrete leukocytosis with no left shift. Computed tomography (CT) scan of the skull evidenced thickening and enhancement of the dura mater in the lateral wall of the right cavernous sinus, erosion of the right petrosal bone and of the clivus. Magnetic resonance imaging (MRI) of the brain showed the same dura mater alteration and extensive impairment of the soft parts at the base of the skull.

\section{Evolution}

Due to the severity of the condition and to the impossibility of excluding an associated neoplastic involvement, material for culture and biopsy was collected through a tympanostomy. The procedure was performed by the otorhinolaryngology team.

The anatomopathological examination revealed a nonspecific inflammatory infiltration, and there was no organism growth at cultures.

Based on the clinical data, the diagnosis of SBO secondary to NOE was made. Antibiotic therapy with ciprofloxacin and intravenous clindamycin was instituted for 10 days, with improvement of pain complaints; next, the treatment was modified to oral ciprofloxacin and sustained for 8 weeks. Facial paresis (House-Brackmann III) partially improved after 6 weeks of antibiotic therapy. Then, the patient was lost to follow-up.

\section{Case 2}

Male patient, 80 years old, admitted with otalgia, otorrhea, and right facial paresis beginning more than 40 days before. Associated dysphagia. Insulin-dependent diabetes mellitus as medical history.

\section{Physical Exam}

Right peripheral facial paralysis (House-Brackmann VI), right IX, X, XI and XII cranial nerve palsy. Otoscopy showed purulent secretion and perforation of the tympanic membrane.

\section{Adjuvant Tests}

A biopsy through tympanostomy was performed, and the secretion was collected for culture. Results showed no signs of neoplasia, and the secretion culture was positive for $P$. aeruginosa.

\section{Evolution}

The patient was treated by the otolaryngology team from another service with intravenous (IV) piperacillin plus tazobactam for 12 weeks, and then with oral ciprofloxacin for 8 weeks. Significant improvement of facial paralysis (HouseBrackmann II) and dysphagia.

After 12 months, the patient returned presenting with left-sided otalgia, headache, dizziness, and left-sided sensorineural hearing loss. At the examination, there was persistent peripheral facial paresis (House-Brackmann III) on the right side. Laboratory tests showed increased inflammatory activity, and a CT scan of the head revealed significant bone destruction around the right jugular foramen, with veiling of mastoid cells. A brain MRI showed enhancement in the middle third of the EAC with bone and soft tissue involvement extending to the right carotid and to the jugular foramina. A gallium 67 scintigraphy was requested, which evidenced radioactive compound accumulation at the right mastoid, extending to the base of the skull and to the contralateral mastoid (the contralateral involvement was not evident in anatomical imaging scans).

The diagnosis of recurrent, bilateral NOE with SBO was made. An indwelling catheter was implanted for home treatment with piperacillin + tazobactam 4.5 g every 8 hours.

The patient presented improvement of the headache, of the otalgia, and of the vertigo and, after 16 weeks, the inflammatory activity was normalized; then, the antibiotic therapy was terminated, and the catheter was removed. 


\section{Case 3}

Male patient, 74 years old, complaining of right otalgia and of temporal headache for 8 months. Initially, he was seen by an otolaryngologist in another service, where, due to the diagnosis of chronic otomastoiditis, he underwent a mastoid biopsy evidencing a chronic inflammatory infiltrate and questionable neoplastic cells. At the time, culture was positive for P. aeruginosa. The patient was treated with systemic corticosteroids, with partial pain relief. He had no history of diabetes mellitus.

Two months after the initial treatment, the patient presented with hoarseness and hypoacusis ipsilateral to the painful symptoms. Three additional biopsies had inconclusive immunohistochemistry results, and a new MRI evidenced progression of the infiltrative process.

The patient was referred to an oncologist who, due to the suspicion of lymphoma, chose to perform chemotherapy with cyclophosphamide, vincristine, and dexamethasone. During treatment, a lumbar puncture showed an increased level of mononuclear cells at the cerebrospinal fluid (CSF), and, then, intrathecal methotrexate was administered.

Due to the worsening of the symptoms, he was referred for evaluation at the neurosurgery service, with an initial suspicion of skull base lymphoma.

\section{Physical Exam}

Right-sided VIII, IX, X, XI and XII cranial nerves dysfunction.

\section{Evolution}

Adjuvant tests and the clinical history were reviewed, with the hypothesis of NOE with evolution for SBO. The treatment was initiated with ciprofloxacin, $400 \mathrm{mg}$ IV every 12 hours for 6 weeks, and then modified to $500 \mathrm{mg}$ (oral) every 12 hours for 8 weeks. The patient presented progressive improvement of the neurological deficits, and, 12 months after the treatment, he had subnormal hearing and mild dysphonia, with no other significant deficits. No signs of relapse at the 6-year follow-up.

\section{Case 4}

Female, 58 years old, with progressively worsening bilateral otalgia for 40 days. Intermittent serous otorrhea for the previous 12 months. Initially treated with amoxicillin and clavulanate for 14 days, with no improvement, and ciprofloxacin, $500 \mathrm{mg}$ (orally) every 12 hours for 10 days, with partial response.

Medical history of systemic arterial hypertension, insulindependent diabetes mellitus, amenorrhea at age 35, and prolactin dosage of $2,000 \mathrm{ng} / \mathrm{mL}$ at a previous examination, without further investigation.

\section{Physical Exam}

Bilateral sensorineural hearing loss (positive Rinne test and bilateral shortening at centralized Weber test), without other focal neurological deficits. Otoscopy showed bilateral serous secretion and a perforation at the anteroinferior quadrant of the right tympanic membrane.

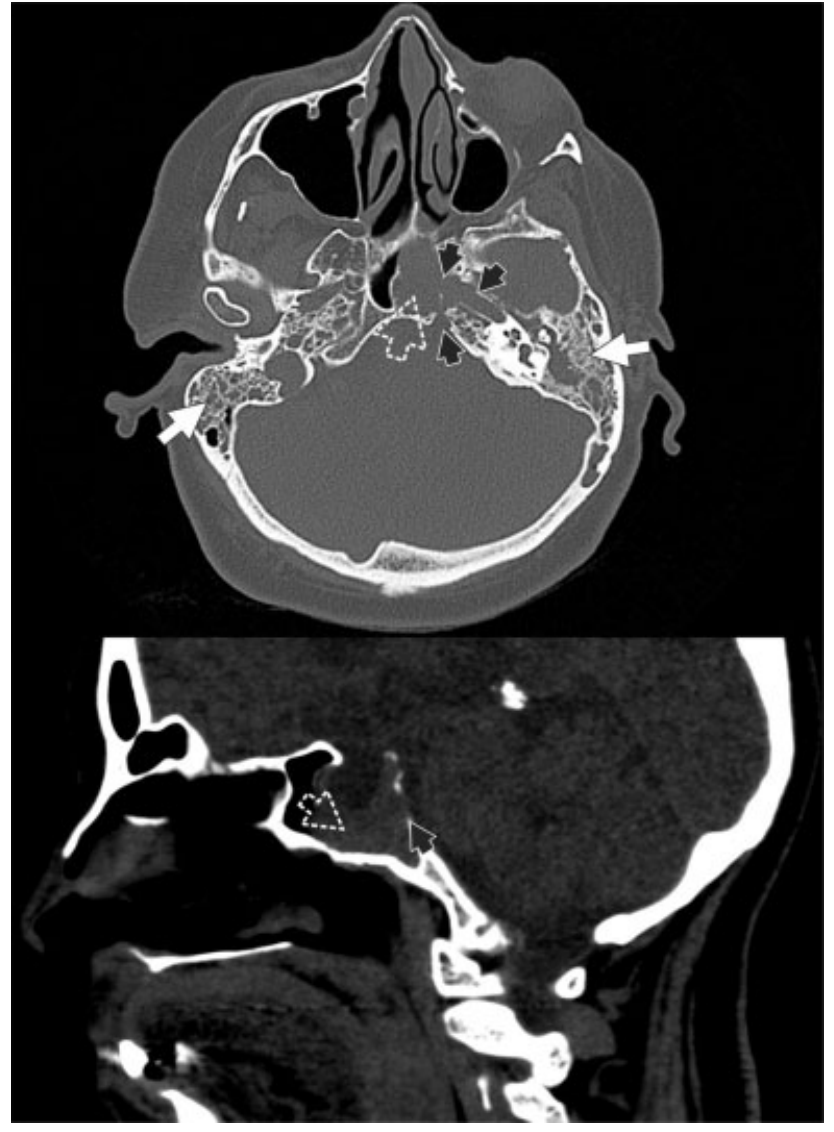

Fig. 1 Computed tomography of the head, axial section, above, and sagittal section, below, case number four. Bilateral mastoid cells (white arrows), erosion of the petrous apex, of the sphenoid sinus wall, of the clivus and of the left carotid canal (black arrows). Expansive lesion with soft tissue density in the sphenoid sinus (dotted arrow).

\section{Adjuvant Tests}

A CT of the mastoid showed bilateral cell veiling, and erosion of the petrous apex, of the sphenoid sinus wall, of the clivus, and of the left carotid canal (-Fig. 1).

An MRI of the brain showed an empty saddle sign, a bulky lesion centered on the left cavernous sinus surrounding the left internal carotid artery and extending to the sphenoid sinus, measuring $2.4 \times 2.1 \times 2.5 \mathrm{~cm}$, tangential to the inferior hypophyseal margin and with no well-defined limits between them (-Fig. 2 and $\mathbf{3}$ ).

C-reactive protein: $63.50 \mathrm{mg} / \mathrm{dL}(\mathrm{VR}<10)$.

Prolactin $>2,000.00 \mathrm{ng} / \mathrm{mL}$ (test result above the maximum value detectable by the method even after $1: 10$ dilution).

Endocrinological screening with no other significant changes.

Left ear secretion with no Gram-positive bacteria or growth at cultures.

\section{Evolution}

After hospital admission and case discussion with the clinical medicine and otorhinolaryngology teams, the diagnostic hypothesis of uncomplicated NOE with SBO associated to a prolactin-secreting hypophyseal macroadenoma was established. Treatment started with piperacillin and tazobactam, 


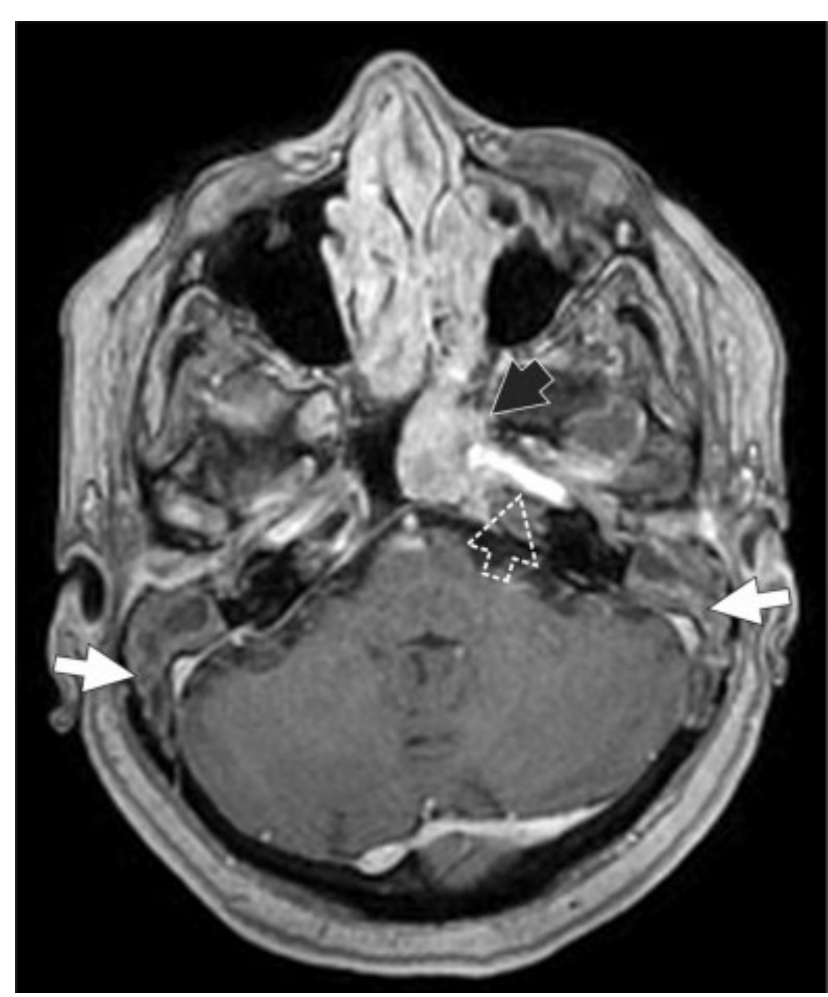

Fig. 2 Magnetic resonance imaging of the brain, axial section, T1-weighted sequence after gadolinium administration, case number four. A large lesion centered at the left cavernous sinus (black arrow) surrounding the left internal carotid artery (dotted arrow), and extending to the sphenoid sinus, measuring $2.4 \times 2.1 \times 2.5 \mathrm{~cm}$. Bilateral complete obliteration of mastoid cells (white arrows).

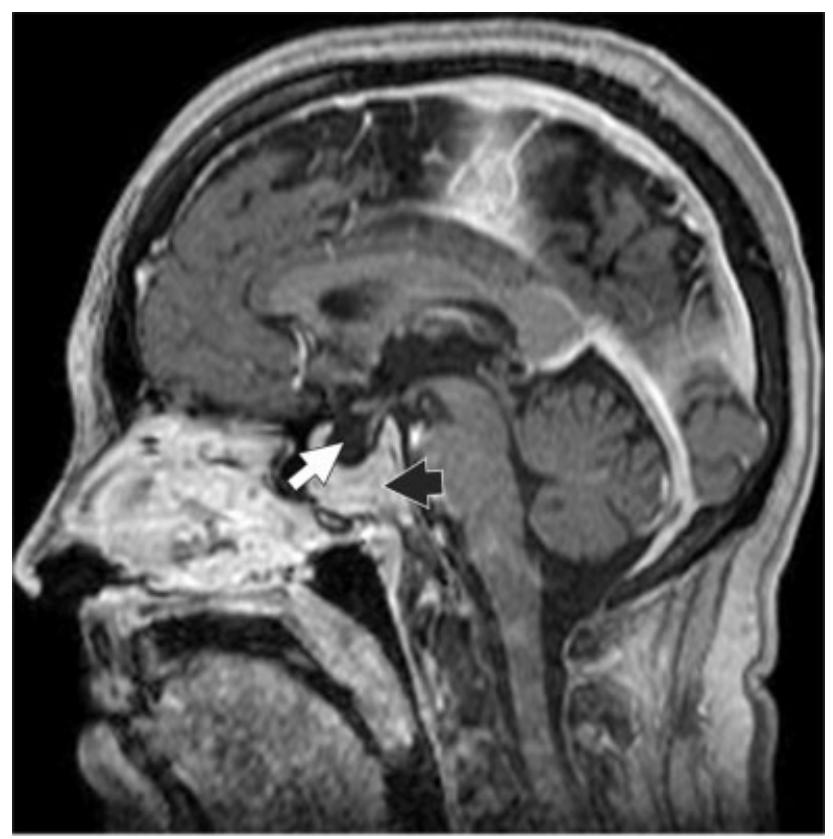

Fig. 3 Magnetic resonance imaging of the brain, midsagittal section, T1-weighted sequence after gadolinium administration, case four. Empty saddle signal (white arrow), massive lesion occupying the sphenoid sinus (black arrow), with homogeneous postcontrast enhancement.
$4.5 \mathrm{~g}$ (IV) every 6 hours and cabergoline. After 7 days, the patient presented with left-sided peripheral facial paresis (House-Brackmann V), and a mastoidectomy was performed for debridement and collection of biopsy and culture material. The procedure was performed by the otorhinolaryngology team (-Fig. 4).

The anatomopathological analysis revealed bone tissue necrosis, with nonspecific inflammatory reaction, and no identifiable fungi or bacteria. No growth or infectious agents were identified at the culture and fungal analysis of the secretion obtained intraoperatively.

Due to worsening during antibiotic therapy and to the absence of identified etiologic agents, the piperacillin and tazobactam regimen was replaced with $2 \mathrm{~g}$ of meropenem every 8 hours for 16 weeks.

The patient presented a partial improvement of the peripheral facial paresis (House-Brackmann IV), along with a persistent auditory deficit and diarrhea secondary to prolonged antibiotic therapy. The prolactin level returned to normal after 3 months of cabergoline administration. A single-photon emission computed tomography (SPECT) with gallium 67 was performed at the end of the IV antibiotic therapy and revealed a discrete isotope concentration at the mastoid bone ( - Fig. 5). Due to the normalization of the evidence of inflammatory activity and of the side effects of the antibiotic therapy, the otorhinolaryngologist decided to discontinue meropenem.

Outpatient follow-up with no clinical signs of relapse within 6 months of treatment.

\section{Discussion}

\section{Clinical Picture}

Persistent headache, otalgia, otorrhea, and cranial nerve deficits (in advanced cases) are the most common changes. This diversity of signs and symptoms makes the patients seek care of several specialties, including neurosurgery. In addition, the diagnosis is difficult, and, on average, it is made 70 days after the onset of symptoms. Chronic otorrhea unresponsive to topical agents, otalgia of difficult management, and irradiation to the temporomandibular joint are the most characteristic findings. At examination, the presence of granulation tissue at the osteocartilaginous junction of the EAC and an intact tympanic membrane are typical. Most cases do not present systemic signs of infection, such as fever and leukocytosis. The progression to SBO affects, in order of frequency, the facial nerve, the glossopharyngeal nerve, the vagus nerve, the spinal root of the accessory nerve, and the hypoglossal nerve. There is no record of optic nerve involvement. ${ }^{4,6}$ Our case series presented clinical symptoms compatible with the literature. Regarding the physical examination, the presence of tympanic perforation in three of the four patients was noted. This finding, not previously described as characteristic, may be justified by the advanced stage at the time of the diagnosis.

\section{Adjuvant Tests}

Culture of EAC secretions or from material obtained through surgical exploration too often results in the isolation of $P$. aeruginosa, but recent studies have shown an up to $70 \%$ 


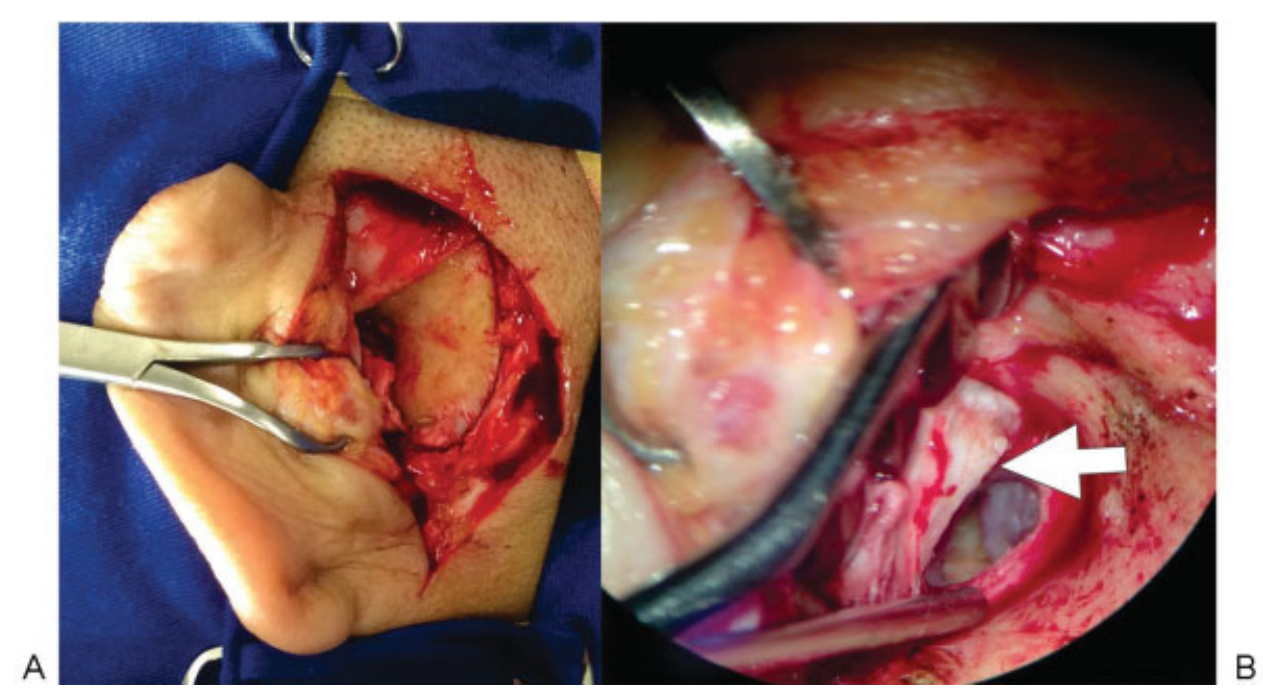

Fig. 4 Intraoperative left-sided mastoidectomy pictures. Retroauricular surgical incision (A). Microscopic view of the facial nerve, $2 \times$ magnification (white arrow), after surgical debridement (B).
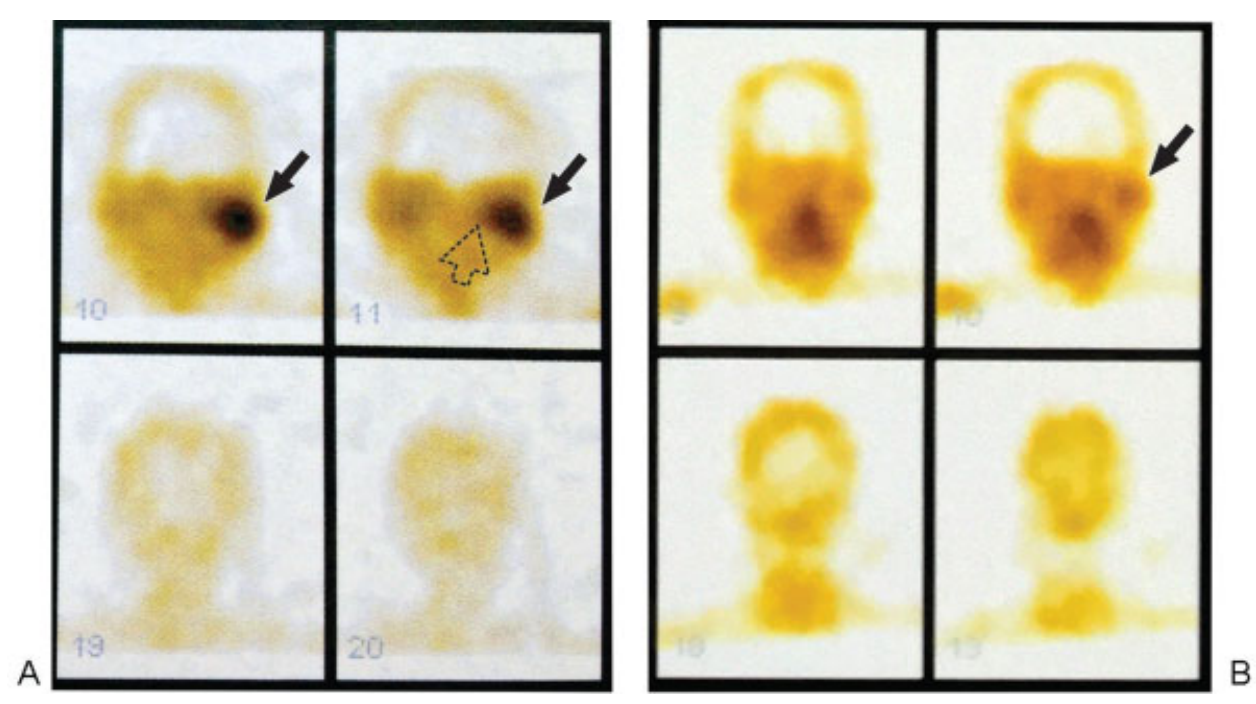

Fig. 5 Single-photon emission computed tomography with gallium 67, coronal sections, before antibiotic therapy (A) and after intravenous antibiotic therapy (B), case number four. (A), Accumulation of the radiopharmaceutical at the left mastoid region (black arrow) and at the left sphenoid and maxillary sinuses topography (dotted arrow). (B), Significant reduction of radiopharmaceutical accumulation compared to the previous examination (A), and persistence of minimal accumulation at the left mastoid bone (black arrow).

negative culture rate. Ideally, collection should be performed prior to the initiation of antibiotic therapy. ${ }^{4,7}$ Therefore, the $50 \%$ positivity rate in the described cases may have been influenced by the prior use of antibiotics with in vitro inhibition of bacterial growth.

The erythrocyte sedimentation rate (ESR) is markedly increased and can be used as a therapeutic response marker, as observed in other osteomyelites. ${ }^{6,8,9}$

There are no sensitivity and specificity studies comparing different imaging tests in NOE diagnosis and therapeutic control, so their use is guided by the technical advantages and deficiencies of each method.

Technetium-99m methylene diphosphonate bone scintigraphy (MDP - Tc 99m) is the most sensitive method; the accumulation of the radiopharmaceutical compound is related to osteoblastic activity. Because it remains altered in an undefined way and in uncomplicated otitis externa cases, this methodology is not a good option for the differential diagnosis and cure control.

Scintigraphy using gallium 67, which is incorporated into granulocytes and bacteria, has a higher specificity compared with bone scintigraphy with MDP - Tc 99m. Some authors advocate that this scintigraphy can be used for cure control and follow-up; however, there is a report of NOE recurrence with normal gallium 67 scintigraphy findings. ${ }^{10}$ Alterations in gallium 67-scintigraphy/SPECT results may precede the bone erosion evidenced in CT by weeks. ${ }^{11}$

Anatomical examinations, such as CT and MRI, allow the accurate topography of the involved area. Computed tomography reveals bone erosion and changes in the adjacent soft parts and in the dura mater (fat blurring, trabecular bone density alteration, and contrast enhancement). Even after satisfactory 
treatment, the eroded areas remain unchanged, which limits the use of CT for treatment control. Magnetic resonance imaging is more sensitive than CT for identifying soft tissue changes (hypersignal in T2-weighted sequences and postcontrast enhancement), as well as in identifying alterations in the lipid content of the bone marrow in NOE cases. ${ }^{4,11,12}$

In the presented cases, nuclear medicine methods for diagnostic purposes were dispensable, since CT revealed bone erosion in all of our patients, suggesting the diagnosis of NOE. In patient number four, scintigraphy with gallium 67 was useful for treatment control after the change in the antibiotic therapy.

Surgical biopsy is the only form of differential diagnosis between squamous cell carcinoma and NOE in cases without
ESR elevation and negative cultures, as imaging findings may be similar. Although rare, there is a description of the coexistence of both conditions in the same patient. ${ }^{13,14}$ It is important to note that, in case number three, whereas the biopsy results showed questionable neoplastic cells, culture was positive for $P$. aeruginosa. In our opinion, this warrants the institution of the antibiotic treatment, since it has a lower risk than the chemotherapy to which the patient was submitted.

\section{Diagnosis}

There is no consensus regarding the diagnostic criteria for NOE, and alterations in adjuvant tests along the history of otalgia, of chronic otorrhea and/or of other symptoms is required in a patient with risk factors. Our sample clearly illustrates the

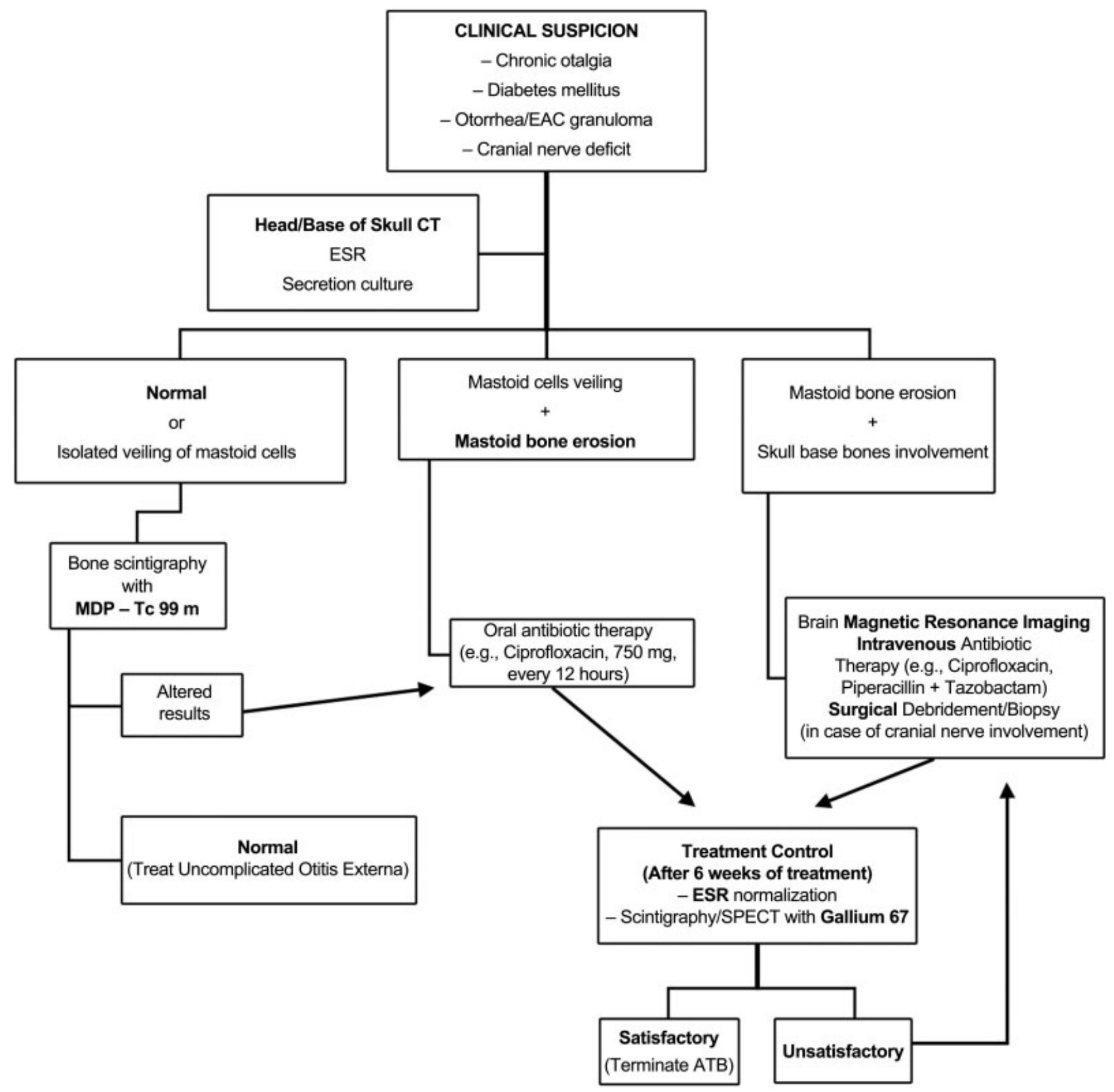

Fig. 6 Flow chart for the diagnosis and treatment of necrotizing otitis externa. Abbreviations: ATB, antibiotic therapy; EAC, external auditory canal; MDP, methylene diphosphonate; SPECT, single-photon emission computed tomography; CT, computed tomography; ESR, erythrocyte sedimentation rate. 
variability of presentations, and it is worth noting that case number three did not present any risk factors for NOE, despite the typical clinical picture. The good response to the clinical treatment can be used for diagnostic confirmation, since the cure rate for NOE is $>75 \%{ }^{4}$

\section{Treatment}

The use of antimicrobial agents with antipseudomonas spectrum is the first choice. Ciprofloxacin, $750 \mathrm{mg}$ orally every 12 hours, shows good bone penetration and high efficacy in NOE treatment when used for at least 6 weeks. Although isolated studies use drugs for $<4$ weeks, there is insufficient scientific evidence to justify this reduction., ${ }^{4,5}$ Intravenous therapy, such as ceftazidime 2 g every 8 hours, is recommended in cases with partial response to oral treatment or progression to SBO. Topical medications elicit no response and distort agent isolation in EAC secretion cultures. ${ }^{4,5}$ Although no agent resistant to conventional antibiotic therapy was documented, due to the unsatisfactory clinical response of case number four, an antibiotic regimen for multi-resistant $P$. aeruginosa (meropenem) was instituted. There is no consensus on the cure criteria that determines discontinuation of antimicrobial therapy, and the recurrence rate is very similar between the various regimens in uncomplicated cases. The last review found an average recurrence rate of $9.7 \%$ in the several series evaluated. ${ }^{4}$

In the rare cases with a fungal etiology (mainly Aspergillus), the prolonged treatment (for $>12$ months) with amphotericin B is recommended.

Antibiotic therapy restricted surgical treatment to the obtainment of materials for culture or biopsy. Debridement is rarely necessary in cases with cranial nerve involvement or secondary abscesses treatment. As the neural lesion is caused by the cytotoxic effect of the inflammatory response, the decompression of the isolated cranial nerves without debridement is not indicated. ${ }^{4-6,15,16}$ Surgical biopsies were performed in all of the cases presented, and a surgical debridement was only required in the last one due to a new cranial nerve deficit during antibiotic therapy.

As previously shown, there is no pathognomonic criterion for the diagnosis of NOE or of its complications, such as SBO. ${ }^{4,17,18}$ The insidious progression and difficult diagnosis delay treatment, which can lead to complications and even death. ${ }^{4,5,8,9}$ As such, we propose a flowchart, which is currently adopted in our institution and is based on the scientific literature used to prepare the present paper (-Fig. 6).

\section{Conclusion}

Necrotizing otitis externa is well-documented in the otorhinolaryngological literature, but poorly described in neurosurgical publications. It is a disease with high morbidity, but treatable. A high index of suspicion, the knowledge of adjuvant diagnostic methods, and early treatment reduce the time to diagnosis and avoid complications. Due to the differential diagnosis with skull base tumors and the possible involvement of neural structures, neurosurgeons must be aware of this condition for an early and adequate treatment, in addition to avoid unnecessary surgical procedures.

Conflicts of Interests

The authors have no conflicts of interests to declare.

\section{References}

1 Meltzer P, Kelemen G. Pyocutaneous osteomyelitis of the temporal bone, madible, and zygoma. Laryngoscope 1959;69(10):1300-1316

2 Rubin J, Yu VL, Stool SE. Malignant external otitis in children. J Pediatr 1988;113(06):965-970

3 Rubin J, Yu VL. Malignant external otitis: insights into pathogenesis, clinical manifestations, diagnosis, and therapy. Am J Med 1988;85(03):391-398

4 Mahdyoun P, Pulcini C, Gahide I, et al. Necrotizing otitis externa: a systematic review. Otol Neurotol 2013;34(04):620-629

5 Ridder GJ, Breunig C, Kaminsky J, Pfeiffer J. Central skull base osteomyelitis: new insights and implications for diagnosis and treatment. Eur Arch Otorhinolaryngol 2015;272(05):1269-1276

6 Rubin Grandis J, Branstetter BF IV, Yu VL. The changing face of malignant (necrotising) external otitis: clinical, radiological, and anatomic correlations. Lancet Infect Dis 2004;4(01):34-39

7 Soudry E, Joshua BZ, Sulkes J, Nageris BI. Characteristics and prognosis of malignant external otitis with facial paralysis. Arch Otolaryngol Head Neck Surg 2007;133(10):1002-1004

8 Verim A, Naiboğlu B, Karaca Ç, Seneldir L, Külekçi S, Oysu Ç. Clinical outcome parameters for necrotizing otitis externa. Otol Neurotol 2014;35(02):371-376

9 Stevens SM, Lambert PR, Baker AB, Meyer TA. Malignant Otitis Externa: A Novel Stratification Protocol for Predicting Treatment Outcomes. Otol Neurotol 2015;36(09):1492-1498

10 Ostfeld E, Aviel A, Pelet D. Malignant external otitis: The diagnostic value of bone scintigraphy. Laryngoscope 1981;91(06):960-964

11 Chakraborty D, Bhattacharya A, Kamaleshwaran KK, Agrawal K, Gupta AK, Mittal BR. Single photon emission computed tomography/computed tomography of the skull in malignant otitis externa. Am J Otolaryngol 2012;33(01):128-129

12 Kwon BJ, Han MH, Oh SH, Song JJ, Chang KH. MRI findings and spreading patterns of necrotizing external otitis: is a poor outcome predictable? Clin Radiol 2006;61(06):495-504

13 Grandis JR, Hirsch BE, Yu VL. Simultaneous presentation of malignant external otitis and temporal bone cancer. Arch Otolaryngol Head Neck Surg 1993;119(06):687-689

14 Mattucci KF, Setzen M, Galantich P. Necrotizing otitis externa occurring concurrently with epidermoid carcinoma. Laryngoscope 1986;96(03):264-266

15 Singh A, Al Khabori M, Hyder MJ. Skull base osteomyelitis: diagnostic and therapeutic challenges in atypical presentation. Otolaryngol Head Neck Surg 2005;133(01):121-125

16 Djalilian HR, Shamloo B, Thakkar KH, Najme-Rahim M. Treatment of culture-negative skull base osteomyelitis. Otol Neurotol 2006; 27(02):250-255

17 Peleg U, Perez R, Raveh D, Berelowitz D, Cohen D. Stratification for malignant external otitis. Otolaryngol Head Neck Surg 2007;137 (02):301-305

18 Illing E, Zolotar M, Ross E, Olaleye O, Molony N. Malignant otitis externa with skull base osteomyelitis. J Surg Case Rep 2011;2011 (05):6 